\title{
Stimulated Brillouin scattering during electron gyro-harmonic heating at EISCAT
}

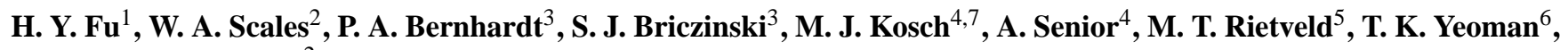 \\ and J. M. Ruohoniemi ${ }^{2}$ \\ ${ }^{1}$ Key Laboratory for Information Science of Electromagnetic Waves (MoE) and School of Information Science and \\ Technology, Fudan University, Shanghai, China \\ ${ }^{2}$ Bradley Department of Electrical and Computer Engineering, Virginia Tech, Blacksburg, USA \\ ${ }^{3}$ Plasma Physics Division, Naval Research Laboratory, Washington, DC, USA \\ ${ }^{4}$ Department of Physics, Lancaster University, Lancaster, UK \\ ${ }^{5}$ EISCAT Scientific Association, Ramfjordmoen, Norway \\ ${ }^{6}$ Department of Physics and Astronomy, University of Leicester, Leicester, UK \\ ${ }^{7}$ South African National Space Agency, Hermanus, South Africa
}

Correspondence to: H. Y. Fu (fuhaiyang@gmail.com)

Received: 15 August 2014 - Revised: 16 June 2015 - Accepted: 24 July 2015 - Published: 11 August 2015

\begin{abstract}
Observations of secondary radiation, stimulated electromagnetic emission (SEE), produced during ionospheric modification experiments using ground-based, highpower, high-frequency (HF) radio waves are considered. The High Frequency Active Auroral Research Program (HAARP) facility is capable of generating narrowband SEE in the form of stimulated Brillouin scatter (SBS) and stimulated ion Bernstein scatter (SIBS) in the SEE spectrum. Such narrowband SEE spectral lines have not been reported using the European Incoherent Scatter (EISCAT) heater facility before. This work reports the first EISCAT results of narrowband SEE spectra and compares them to SEE previously observed at HAARP during electron gyro-harmonic heating. An analysis of experimental SEE data shows observations of emission lines within $100 \mathrm{~Hz}$ of the pump frequency, interpreted as SBS, during the 2012 July EISCAT campaign. Experimental results indicate that SBS strengthens as the pump frequency approaches the third electron gyro-harmonic. Also, for different heater antenna beam angles, the CUTLASS radar backscatter induced by HF radio pumping is suppressed near electron gyro-harmonics, whereas electron temperature enhancement weakens as measured by EISCAT/UHF radar. The main features of these new narrowband EISCAT observations are generally consistent with previous SBS measurements at HAARP.
\end{abstract}

Keywords. Ionosphere (active experiments; particle acceleration; plasma waves and instabilities)

\section{Introduction}

Ionospheric plasma turbulence can be created by injection of powerful high-frequency (HF) radio waves from groundbased transmitters. The interaction between high-power electromagnetic waves and plasmas in the ionosphere can produce stimulated electromagnetic emissions (SEEs), first reported by Thidé et al. (1982) and reviewed by Leyser (2001). SEE spectral lines in the scattered wave can be utilized to remotely probe the properties of the ionosphere as well as actively study radio pump-induced phenomena such as artificial airglow during modification of the ionosphere (e.g. Bernhardt et al., 2009, 2010; Pedersen et al., 2010; Mahmoudian et al., 2013a).

Wideband SEE within $100 \mathrm{kHz}$ of the pump frequency has been studied extensively for several decades. However, due to updates of the HAARP facility in 2007, it has been possible to investigate narrowband (within roughly $1 \mathrm{kHz}$ of the pump frequency) SEE near the resonance altitude in recent years. Stimulated Brillouin scattering (SBS) has been recently observed in the high-power, HF wave ionospheric experiments, shifted by a few tens of hertz from the pump 
frequency (Norin et al., 2009). During the past few years, SEE observations at HAARP have revealed a plethora of narrowband SEE lines associated with SBS and stimulated ion Bernstein scatter (SIBS) (e.g. Norin et al., 2009; Bernhardt et al., 2009, 2010; Samimi et al., 2012, 2013, 2014). Fu et al. (2013) further investigated SBS and SIBS and their interrelationship in detail over a range of aspect beam angles and frequency stepping near electron gyro-harmonics. Mahmoudian et al. (2013b) investigated the threshold for SBS at HAARP and experimentally showed that an effective radiated power $(\mathrm{ERP}) \sim 140 \mathrm{MW}$ is required to excite SBS associated with ion acoustic (IA) waves. These observations motivated this campaign at EISCAT to produce SBS using lower-power HF heating.

The physical process of SBS involves a nonlinear interaction in which an incident (pump) electromagnetic wave decays into an electrostatic IA wave and a scattered electromagnetic wave via the Brillouin instability. In such threewave interaction processes, the wave-matching conditions are satisfied: $\omega_{0}=\omega_{\mathrm{S}}+\omega_{\mathrm{L}}$ and $\boldsymbol{k}_{0}=\boldsymbol{k}_{\mathrm{S}}+\boldsymbol{k}_{\mathrm{L}}$, where $\omega$ is the wave frequency; $\boldsymbol{k}$ is the wave propagation vector; and the subscripts $0, S$ and $L$ denote the pump waves, the scattered waves and low-frequency waves, respectively. Such laser-induced parametric decay SBS processes have been commonly detected and thoroughly studied in unmagnetized plasmas as summarized by Kruer (1988). The first SBS decay process has been detected only recently in high-power, HF ionospheric modification experiments.

Observations of SBS at HAARP were considered to arise from the plasma reflection resonance height where $\omega_{0} \approx \omega_{\mathrm{p}}$ and the upper hybrid UH resonance height $\omega_{0} \approx \omega_{\text {uh }}$. However, for underdense plasmas, $\omega_{0} \gg \omega_{\mathrm{p}}$, it has been suggested that SBS may be produced using the EISCAT incoherent radar facility as first discussed by Dysthe et al. (1977). A modification in the double-humped spectra of incoherent backscatter was predicted by Fejer (1977) at Jicamarca and Arecibo. Experimental observation of SBS using the Jicamarca $50 \mathrm{MHz}$ incoherent scatter radar can cause asymmetry as large as $25 \%$ in the incoherent ionic backscatter spectrum (Fejer et al., 1978), resulting in errors of $10-15 \mathrm{~m} \mathrm{~s}^{-1}$ in the measured velocity.

The primary purpose of the experiment during the 2012 EISCAT campaign was to investigate the possibility of generating SBS using the EISCAT HF heating facility and its modification effects using simultaneous incoherent EISCAT/UHF radar and CUTLASS HF radar diagnostics. It was also the intention to study the correlation between SEE, field-aligned irregularities (FAIs) and electron temperature enhancement near the electron gyro-harmonic frequency for different aspect angles during the ionospheric modification experiment. This paper is organized as follows. In the next section, experimental procedure and diagnostics are described. Thereafter, the experimental observations and analysis are given. Finally, a summary and conclusions are provided.

\section{Experiment setup}

The EISCAT HF facility $\left(69.59^{\circ} \mathrm{N}, 19.23^{\circ} \mathrm{E}\right)$ near Troms $\varnothing$, in northern Norway (Rietveld et al., 1993), was used to produce SEE during a campaign on 3-10 July 2012. The HF transmitter was operated at O-mode polarization with full power. The pump frequency was stepped upward and downward through the third harmonic of the ionospheric electron gyro-frequency $3 f_{c e}$. The pump frequency steps every $20 \mathrm{kHz}$ in a range of $3.9 \mathrm{MHz} \leq f_{0} \leq 4.2 \mathrm{MHz}$. The heater duty cycle was typically $1 \mathrm{~min}$ on and $1 \mathrm{~min}$ off, unless otherwise stated. All 12 transmitters on array 2 were used at $80 \mathrm{~kW}$ each, resulting in a gain of $22.4 \mathrm{dBi}$ and effective radiated power (ERP) of approximately $148 \mathrm{MW}$. The beam angle was scanned in small $6^{\circ}$ steps in the magnetic meridian. For each angle, the heating time period was $30 \mathrm{~min}$ during the frequency stepping cycle.

The SEE receiver was installed near Breivikeidet, Norway $\left(69.64^{\circ} \mathrm{N}, 19.49^{\circ} \mathrm{E}\right)$, about $13 \mathrm{~km}$ east-northeast of the EISCAT site. The antenna was a broadband resistively loaded folded dipole. The receiver was an Ettus Research USRP N210 fitted with a GPS-disciplined oscillator to provide precise time and frequency references. The receiver was tuned to $6 \mathrm{MHz}$ and recorded at $6.25 \mathrm{MHz}$ sample rate to cover all heater frequencies. The sampled data are processed with the fast Fourier transform (FFT) to yield low-frequency spectra.

The EISCAT $931 \mathrm{MHz}$ UHF radar was operated in Beata mode, which enables measurement from 50 to $700 \mathrm{~km}$ with a minimum of $5 \mathrm{~s}$ time resolution and $3.5 \mathrm{~km}$ range resolution. The UHF radar data were integrated for $60 \mathrm{~s}$ to reduce measurement error, with approximately $14 \mathrm{~km}$ resolution near the reflection region. The UHF incoherent scatter radar can provide ionospheric electron temperature, electron density, plasma/ion-line frequency spectra and the reflection altitude. A dynasonde, co-located with EISCAT, made a sounding every $6 \mathrm{~min}$, which can also provide the electron density profile and the reflection altitude in the ionosphere.

The electron gyro-harmonic effects of SEE are associated with HF, pump-induced FAIs, consisting of narrow filaments of density depletions a few percent in amplitude and elongated several tens of kilometres along the geomagnetic field (Fialer, 1974). The heater produced FAIs were diagnosed by CUTLASS HF coherent radars at Hankasalmi, Finland, and Thykkvibær, Iceland. The CUTLASS pair of HF radars in the Northern Hemisphere is part of the SuperDARN network of HF coherent radars, which is a frequency-agile bistatic HF radar system operating in the range $8-20 \mathrm{MHz}$ (Robinson et al, 1997). During this experiment, the CUTLASS radars operated in "stereo" mode by utilizing some of the radar's spare duty cycle. The radar employed three frequency bands - 9$10,13-14$ and $16-17 \mathrm{MHz}$ - which are sensitive to FAIs with spatial sizes of between 8 and $17 \mathrm{~m}$. The dwell (integration) time on each radar beam is $1 \mathrm{~s}$ for Hankasalmi. 

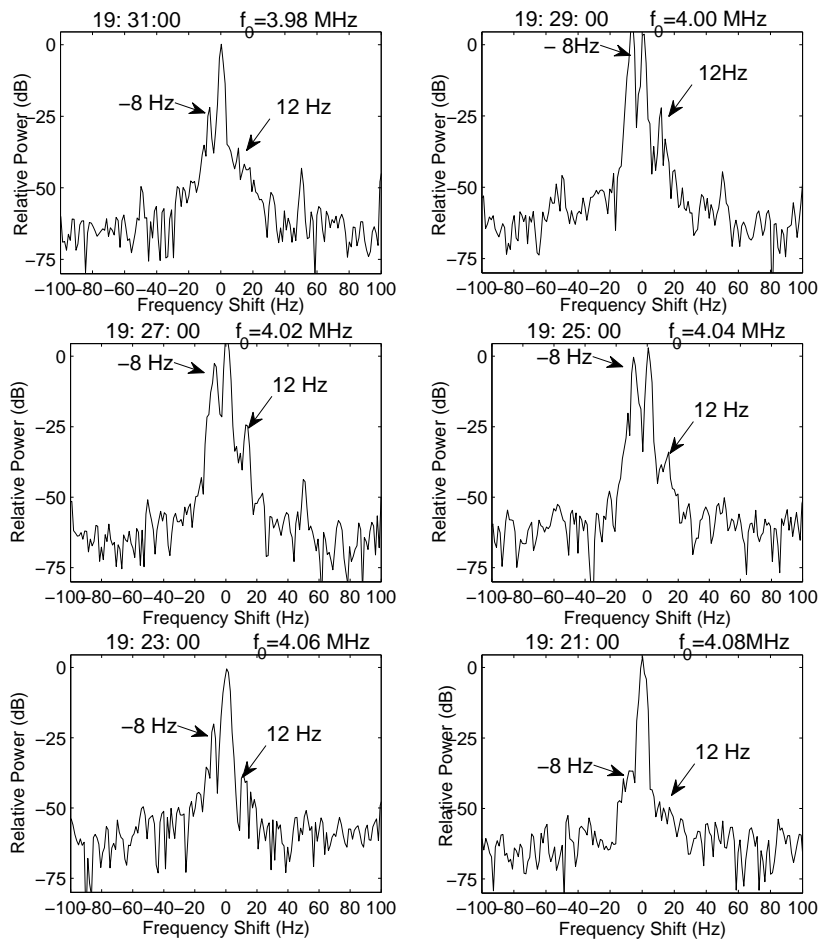

Figure 1. Narrowband SEE frequency spectra of HF scattered signals showing strong emission lines at $8-12 \mathrm{~Hz}$ using the EISCAT $\mathrm{HF}$ transmitter operating at varying pump frequencies near $3 f_{c \mathrm{e}}$ during 19:20-19:32 UT on 3 July 2012. The heating beam points towards the magnetic zenith direction with $1 \mathrm{~min}$ on/off duty cycle.

\section{Experimental results}

\subsection{Narrowband SEE observations}

Figure 1 shows narrowband frequency spectra of the scattered HF pump wave for heating near the third electron gyroharmonic frequency, $3 f_{c e}$, during 19:20-19:32 UT on 3 July 2012. Electromagnetic backscattered waves were produced by the EISCAT HF transmitter operating with an ERP of $148 \mathrm{MW}$ for the magnetic zenith beam. Dynasonde data at 19:28 UT on 3 July 2012 indicate quiet ionospheric status. The reflection altitude for the pump frequency $4.04 \mathrm{MHz}$ is $\sim 215 \mathrm{~km}$ according to dynasonde data. Strong emissions downshifted by $\sim 8 \mathrm{~Hz}$ and upshifted by $\sim 12 \mathrm{~Hz}$ in the spectra are clearly observed in Fig. 1 with power within $10 \mathrm{~dB}$ relative to the reflected pump wave. The power of the downshifted (or Stokes) emission line is larger than the upshifted (anti-Stokes) emission.

These shifted spectral lines observed in Fig. 1 show a similar frequency shift and relative amplitude of Stokes and antiStokes lines when compared to experimental observations at HAARP (e.g. Norin et al., 2009; Bernhardt et al., 2009). The reflected pump waves and scattered electromagnetic waves combine to produce upshifted SBS lines with lower intensity and slightly higher $4-5 \mathrm{~Hz}$ frequency offset than the down-
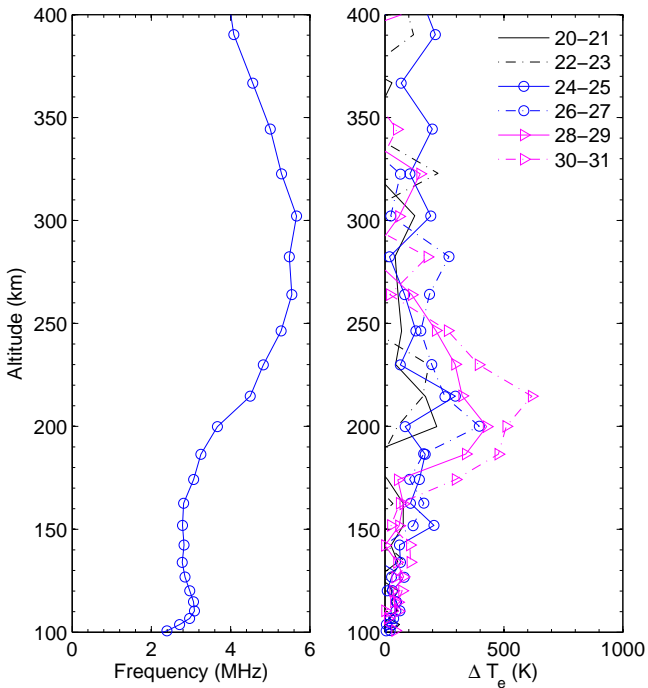

Figure 2. The measured UHF radar plasma frequency and electron temperature enhancement $\Delta T_{\mathrm{e}}$ during 19:24-19:30 UT on 3 July 2012. Note that the reflection altitude is approximately $215 \mathrm{~km}$ for the pump frequency $4.04 \mathrm{MHz}$.

shifted SBS. Upshifted SBS lines have been previously explained as follows (Bernhardt et al., 2009). After the upward pump reflects near-zero refractive index, yielding a downward pump wave, it scatters with IA waves to produce another upward electromagnetic wave with wave vector $\boldsymbol{k}_{0}=\boldsymbol{k}_{\mathrm{S}}+\boldsymbol{k}_{\mathrm{L}}$ and $\boldsymbol{\omega}_{0}=\omega_{\mathrm{S}}+\boldsymbol{\omega}_{\mathrm{L}}$. Bernhardt et al. (2010) interpreted $\Delta f_{\mathrm{IA}}=-\left(f-f_{0}\right)=6 \sim 12 \mathrm{~Hz}$ below/above the pump frequency $f_{0}$ as SBS from the plasma resonance region. Theoretical and experimental works on SBS indicate that the production of downshifted lines should be preferred.

The strength of observed sideband emissions in Fig. 1 depends on the electromagnetic pump wave frequency as well. During the frequency stepping, the SBS emissions were observed at pump frequencies $4.04,4.02$ and $4.00 \mathrm{MHz}$, relatively close to $3 f_{c \mathrm{ce}}$. The frequency dependence of the emission may be attributed to the EISCAT HF transmitter power being near the threshold for excitation. It may be postulated that, when less anomalous absorption occurs near $3 f_{c e}$, more heater power can be transmitted to a higher resonance altitude where SBS occurs. Anomalous absorption is due to scattering of the electromagnetic waves on FAI with a wide spatial spectrum. For pump frequency near $n f_{c \mathrm{e}}(n=3,4)$, FAI intensity and anomalous absorption are minimum (see Leyser, 2001, and references therein). This will be discussed further in the next section.

Figure 2 shows the measured UHF radar plasma frequency $\omega_{\mathrm{p}}$ and electron temperature enhancement $\Delta T_{\mathrm{e}}$ profile vs. height during 19:20-19:32 UT on 3 July 2012. The integration time is $60 \mathrm{~s}$. Incoherent radar data indicate that the reflection altitude is $215 \mathrm{~km}$ for the pump frequency $4.04 \mathrm{MHz}$. The electron temperature enhancement at $\sim 215 \mathrm{~km}$ min- 

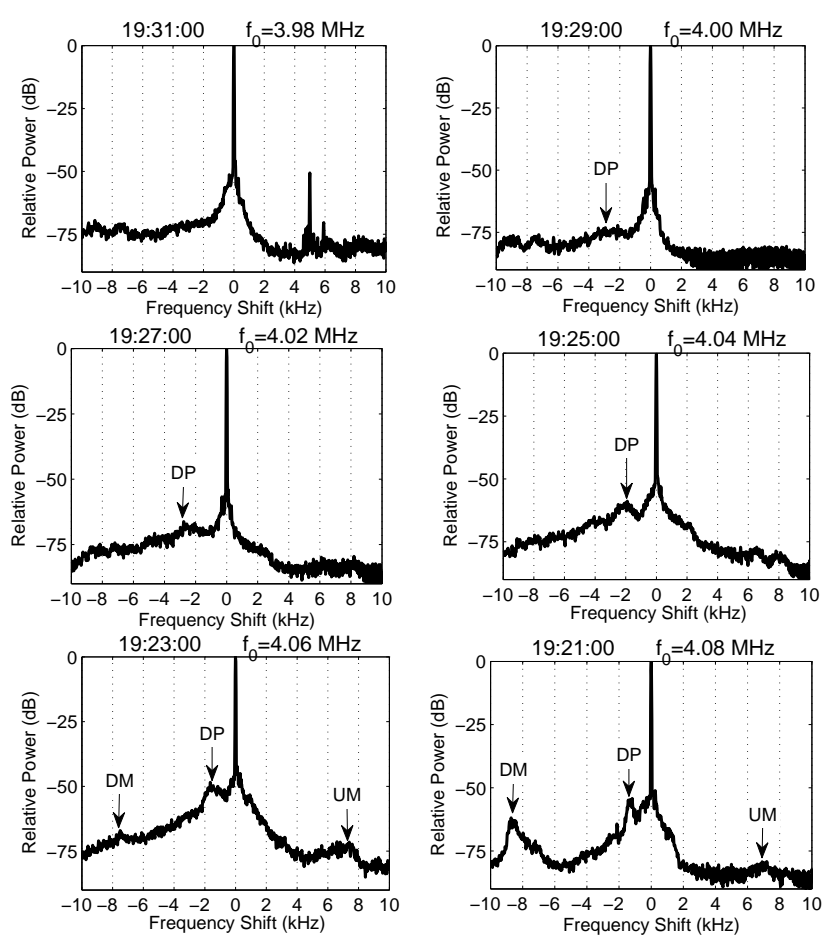

Figure 3. Wideband SEE frequency spectra of HF scattered signals from the EISCAT HF transmitter operating at varying pump frequencies near $3 f_{c e}$. The heating direction is along the magnetic zenith. According to DP measurement, $f_{0} \approx 3 f_{c \mathrm{e}}$ occurs between 4.04 and $4.06 \mathrm{MHz}$.

imizes for pump frequency $4.04 \mathrm{MHz}$ during the interval 19:24-19:25 UT on 3 July 2012. The electron temperature enhancement and anomalous absorption are correlated as observed by Honary et al. (1995). Electron temperature and anomalous absorption are minimized when pumping on a gyro-harmonic frequency because the growth of small-scale $(1-10 \mathrm{~m})$ field-aligned striations is suppressed, as will be discussed in further detail in the next section. Based on incoherent scatter radar data, the pump-induced electron temperature enhancement reaches approximately $500-600 \mathrm{~K}$ for the pump frequency $3.98 \mathrm{MHz}$ in the heated region in Fig. 2.

The wave-matching condition for SBS is $\boldsymbol{k}_{\mathrm{L}} \cong 2 \boldsymbol{k}_{0}$ (Bernhardt et al., 2010). An analytical expression for the IA waves, propagating with an angle $\theta$ to the ambient magnetic field, can be expressed as $\omega_{\text {IA }}=$ $\sqrt{\left(k_{\mathrm{IA}}^{2} c_{\mathrm{IA}}^{2} \cos ^{2} \theta\right) /\left(1+k_{\mathrm{IA}}^{2} c_{\mathrm{IA}}^{2} / \Omega_{c \mathrm{i}}^{2}\right)}$ when the ion sound waves have wavelengths much larger than a Debye length $k_{\mathrm{IA}} \lambda_{\mathrm{d}} \ll 1$. Here, $\Omega_{c \text { i }}$ is the ion gyro-frequency and $c_{\mathrm{IA}}=$ $\sqrt{\left(\gamma_{\mathrm{e}} T_{\mathrm{e}}+\gamma_{\mathrm{i}} T_{\mathrm{i}}\right) / m_{\mathrm{i}}}$ is the IA velocity with $\gamma_{\mathrm{e}}=1$ and $\gamma_{\mathrm{i}}=$ $3, T_{\mathrm{e}}$ and $T_{\mathrm{i}}$ are the electron and ion temperature, respectively, and $\lambda_{\mathrm{d}}$ is the Debye length (Bernhardt et al., 2009). The ion gyro-frequency representative of the conditions over EISCAT at $215 \mathrm{~km}$ is estimated to be $f_{c \mathrm{i}} \approx 46.0 \mathrm{~Hz}$. Based on incoherent scatter radar data, the electron temperature is taken to be $T_{\mathrm{e}}=2600 \mathrm{~K}$ and $T_{\mathrm{e}} / T_{\mathrm{i}}=2.5$. The wave- matching condition predicts that the strongest IA wave emissions $f_{1} \sim 8 \mathrm{~Hz}$ are excited near the reflection resonance altitude where the local plasma frequency becomes close to the pump frequency.

\subsection{Associated wideband SEE and irregularities}

A classic feature of the steady-state SEE spectrum is the downshifted peak (DP) when pumping near electron gyroharmonics (Leyser, 2001). The DP is located at $\Delta f_{\mathrm{DP}} \approx 1-$ $3 \mathrm{kHz}$ below the pump frequency. An upshifted peak (UP) feature occurs above the pump frequency at approximately the mirror frequency of the DP. The DP, 2DP and UP can be simultaneously observed when the pump frequency approaches the $n$th $(n \geq 3)$ electron gyro-harmonic frequency $n f_{c \text { e }}$. Stubbe and Kopka (1990) stated that the DP has been found to be a strong feature for $f_{0}=3 f_{c \text { e }}$ and weak sign for $f_{0}=4 f_{c \mathrm{e}}, 5 f_{c \mathrm{e}}$. It is worth mentioning that Mahmoudian et al. (2013a) recently noted a similar spectral feature for $f_{0} \approx$ $2 f_{c \text { ce }}$ pumping with frequency offset $\Delta f \approx 500-1000 \mathrm{~Hz}$.

Figure 3 shows the dependence of wideband SEE features on the pump frequency when pumping near $3 f_{c e}$ for the same time period and experimental conditions as the narrowband SEE in Fig. 1. For pump frequencies close to $4.04 \mathrm{MHz}$, the DP at approximately $\sim 2 \mathrm{kHz}$ below the pump frequency develops. The DP frequency offset drops from approximately -2.5 to $-1.6 \mathrm{kHz}$ as the pump frequency approaches $3 f_{c e}$, consistent with previous experimental observations (Stubbe et al., 1994). The DP serves as a good indicator for the pump frequency close to the third electron gyro-harmonic frequency (Stubbe and Kopka, 1990; Stubbe et al., 1994; Honary et al., 1995). The DP frequency offset for the pump frequency near $3 f_{c e}$ can be approximately estimated based on existing theoretical models (Huang and Kuo, 1995; Hussein and Scales, 1997; Mahmoudian et al., 2013a). A detailed description of these DP models is beyond the scope of the current paper and will be pursued in future works. If the pump frequency increases further above electron gyro-harmonic, the downshifted maximum (DM) spectral line (Leyser, 2001) at approximately $8-8.5 \mathrm{kHz}$ below the pump frequency appears in the lower sideband spectrum. The DM involves electrostatic lower hybrid waves, where the lower hybrid wave frequency is estimated to be $\omega_{\mathrm{lh}} \simeq 7.5 \mathrm{kHz}$. The presence of a DM and upshifted maximum (UM) in the SEE spectrum, which is closely correlated with FAIs, can also serve as a indicator of whether the pump frequency is near to or far from a harmonic of the electron gyro-frequency (Leyser et al., 1994). Another set of repeated daytime experiments shows the dependence of the DP on the pump frequency for different beam angles. The behaviour at different angles is similar to the magnetic zenith case. If the transmitter beam angle is tilted further off the magnetic field line, the amplitude of the DP becomes weak and the second downshifted peak (2DP) and UP may not appear in the spectra. 

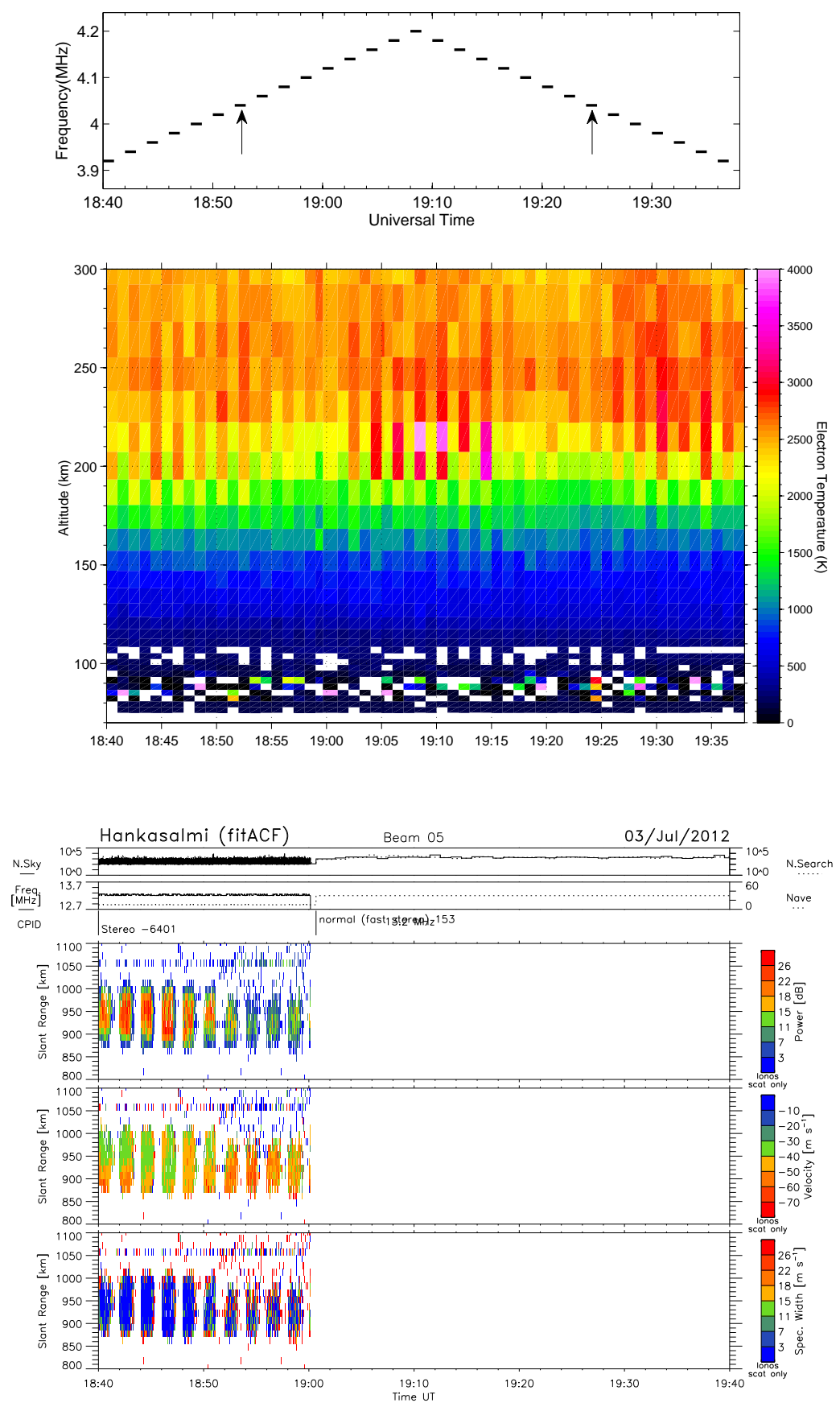

Figure 4. The results of HF pumping during 18:40-19:40 UT on 3 July 2012. The upper panel shows the pump frequency, where the black arrows indicate the pump frequency $4.04 \mathrm{MHz}$ (approximately $3 f_{c e}$ ) at 18:52-18:53 and 19:14-19:15 UT. The middle panel shows the electron temperature profile measured by the EISCAT UHF radar with the integration time $60 \mathrm{~s}$. The lower panel shows CUTLASS backscatter power, Doppler velocity, and spectral width vs. slant range (line-of-sight distance) in the heating region over EISCAT.

Figure 4 depicts the HF pumping frequency scheme, the electron temperature profile measured by the EISCAT UHF radar and CUTLASS backscatter power, the Doppler velocity, and the spectral width during 18:40-19:38 UT on 3 July 2012. The upper panel shows the pump frequencies between 3.92 and $4.2 \mathrm{MHz}$. The arrows indicate the pump frequency near $3 f_{c e}$ (i.e. $f_{0}=4.04 \mathrm{MHz}$ ). The middle panel shows the electron temperature measured by the EISCAT $931 \mathrm{MHz}$ UHF incoherent scatter radar. For the upward frequency stepping, the electron temperature enhancement minimizes during 18:54-18:55 $\left(f_{0}=4.06 \mathrm{MHz}\right)$ and $18: 56-18: 57\left(f_{0}=4.08 \mathrm{MHz}\right)$. For the downward frequency 
stepping, the electron temperature enhancement reduces during 19:22-19:23 $\left(f_{0}=4.06 \mathrm{MHz}\right)$ and $19: 24-19: 25\left(f_{0}=\right.$ $4.04 \mathrm{MHz}$ ), which correspond to strong DP as observed in Fig. 3. The electron temperature enhancement minimizes when the pump frequency approaches $3 f_{c e}$. This agrees with previous experimental observations (Honary et al., 1995).

The electron temperature enhancement exhibits an asymmetry for pump frequencies above and below $3 f_{c e}$ in the middle panel of Fig. 4. It should be noted that the measurement error increases during 19:08-19:15 for $f_{0}>3 f_{c e}$ in the narrow altitude range close to the heater reflection height, since the electron temperature retrieval algorithm is based on the ion-line spectra which are modified by HF pump-induced effects. It is unclear whether there is more efficient electron heating for $f_{0}>3 f_{c \mathrm{e}}$ from these observations. Further analysis will be required on the simultaneous ion-line spectra and SEE measurements.

The lower panel shows the CUTLASS backscatter power, Doppler velocity, and spectral width from beam 5 of the Hankasalmi radar. The backscattered signals are produced by Bragg scattering of the sounding waves from pre-existing or pump-induced field-aligned striations. The aspect-angle dependence for scattering requires that the radio wave $\boldsymbol{k}$ vector be close to orthogonal to the magnetic field $\mathbf{B}$. The CUTLASS radar measures F-region irregularities with a plasma $\mathbf{E} \times \mathbf{B}$ drift $v_{\mathrm{d}}$. The HF radar beam that is pointed to magnetic north measures an eastward (zonal) electric field orthogonal to $\mathbf{B}$. The radar cycles through three frequencies: 9.9, 13.2 and $16.6 \mathrm{MHz}$. Only $13.2 \mathrm{MHz}$ corresponds to strong backscatter from FAIs of wavelength $\sim 11 \mathrm{~m}$. When the pump frequency approaches $4.04 \mathrm{MHz}$ during the time period 18:52-18:53, there is a clear reduction observed in the backscatter power from a peak of $30 \mathrm{~dB}$ to approximately $15 \mathrm{~dB}$. There exists a minimum of the backscatter power during the time periods $18: 54-18: 55\left(f_{0}=4.06 \mathrm{MHz}\right)$ and 18:56-18:57 $\left(f_{0}=4.08 \mathrm{MHz}\right)$. The minimum backscatter power exactly corresponds to the minimum electron temperature enhancement. Based on the temporal evolution of the backscatter power between approximately 0 and $25 \mathrm{~dB}$, the rise time of FAIs is estimated to be less than $10 \mathrm{~s}$ and the decay time is approximately $40 \mathrm{~s}$.

The Doppler velocity during the HF pumping is due to the $\mathbf{E} \times \mathbf{B}$ drift of HF-induced FAIs and reaches a maximum value of approximately $-50 \mathrm{~m} \mathrm{~s}^{-1}$ corresponding to a frequency of approximately $5 \mathrm{~Hz}$. The spectral width is typically less than $5 \mathrm{~m} \mathrm{~s}^{-1}$.

Unfortunately, after 19:00 UT in Fig. 4, the CUTLASS radar was switched back to operate in its standard mode. When pumping above $3 f_{c e}$ after 19:00, the spectral width may vary but is not observed in these measurements. In summary, these observations indicate that when the electron temperature is reduced and FAIs become weak, both SBS and DP are observed in the scattered signals.

These DP line observations as well as electron temperature and FAIs hold true for different heater beam angles. The FAIs are suppressed when pumping very close to $3 f_{c e}$, resulting in weak CUTLASS backscatter (Honary et al., 1999). While the electron temperature is minimum in correlation with fieldaligned striation suppression, a prominent DP with a small frequency shift is observed in the spectrum. When pumping near electron gyro-harmonics, less absorption occurs near the upper hybrid resonance level, giving rise to the simultaneous presence of a strong DP emission line (Huang and Kuo, 1995). Huang and Kuo (1995) proposed a generation mechanism for the DP and UP emissions through parametric decay of upper hybrid/electron Bernstein (UH/EB) wave into another UH/EB sideband wave and a nearly perpendicularly propagating IA decay mode wave in an altitude region slightly above the double resonance layer. Such a DP generation mechanism process involves short-scale, field-aligned density irregularities $\left(\boldsymbol{k}=\boldsymbol{k}_{0}\right)$ through a thermal oscillating two streaming instability (OTSI) process (Dysthe et al., 1983). This differs from SBS, which does not involve fieldaligned density irregularities in its generation process. The threshold for SBS is usually higher than DP emissions, and DP appears in the spectra almost immediately after the heater is turned on (Mahmoudian et al., 2013a).

\section{Discussion and conclusions}

Using an ERP of $\sim 148 \mathrm{MW}$, the EISCAT HF facility may generate SBS emissions. It is noted that this observed power level is less than that required for SBS generation from the plasma reflection altitude at HAARP $(\sim 320 \mathrm{MW})$ (Mahmoudian et al., 2013b). During this campaign, the IA-related emission lines shifted by $6-12 \mathrm{~Hz}$ from the pump are observed for the pump frequency near the third electron gyroharmonic. Also, the amplitude of the downshifted $\sim 8 \mathrm{~Hz} \mathrm{IA}$ line is larger than the upshifted $\sim 12 \mathrm{~Hz}$ IA line. These spectral characteristics of IA emission lines reported in this paper agree with SBS lines from the plasma resonance region previously observed at HAARP. As for the critical differences, more carefully designed experiments are necessary in the future to make substantive conclusions at this time.

To further investigate SBS generation near the third gyroharmonic, the DP lines are observed simultaneously with electron temperature from EISCAT/UHF data and FAIs from CUTLASS radar. During the frequency stepping, experimental results show that DP structures become prominent as $f_{0}$ approaches $3 f_{c e}$, while FAIs are suppressed and electron temperature becomes minimum. It is postulated that the enhancement of SBS near $3 f_{c e}$ may be explained by weak FAIs, with the result that more power reaches the reflection altitude. The correlations between DP structures, plasma line/ion-line spectrum and FAIs for varying transmitter beam angle have been observed during the experiments and show qualitatively similar behaviour to pumping along the magnetic field but are not presented here. Further examination of the data will be provided in the future. 
Although fundamentally different physical processes, SBS and DP SEE are both a result of parametric decay instabilities with IA waves as the low-frequency decay modes. SBS involves slow magnetosonic IA waves for frequencies below the ion cyclotron frequency, while DP involves IA waves for frequencies above the ion cyclotron frequency. These spectral lines are therefore important consequences of IA waves in the wideband and narrowband SEE spectrum leading to additional diagnostic information of ionospheric conditions. The characteristics of the two processes are compared from the present experimental observations as follows:

1. For pump frequency stepping across electron gyroharmonics, the DP is strengthened as $f_{0}$ approaches $3 f_{c e}$. SBS is also observed to be enhanced as $f_{0}$ approaches $3 f_{c \mathrm{ce}}$. It is postulated that less absorption and consequently more power near $3 f_{c e}$ plays an important role in exciting SBS emissions.

2. The frequency offset of SBS at $8-12 \mathrm{~Hz}$ appears roughly independent of $f_{0}$ where the DP is highly sensitive to $f_{0}$ with $\Delta f$ varying with proximity of $f_{0}$ to $3 f_{c \mathrm{c}}$. When the pump frequency is increased towards $3 f_{c e}$, the reduced frequency offset is explained by the wavematching condition of the upper hybrid/electron Bernstein (UH/EB) parametric decay process (Huang and Kuo, 1995).

3. The SBS from the plasma resonance altitude is considered to be a process which does not depend on the presence of field-aligned striations. This is different from models of the DP emission generation, which involves the existence of FAIs (Huang and Kuo, 1995). The DP may require a lower power threshold field than that for SBS as observed in experiments. Previous experiments have observed DP emissions at $4.04 \mathrm{MHz}$ with an ERP of $86 \mathrm{MW}$ (Stubbe et al., 1984) at EISCAT. According to calculations by Huang and Kuo (1995), the heater nominal power threshold is an ERP of $\sim 12 \mathrm{MW}$ by ignoring D-region absorption, a factor of 10 less than the threshold for SBS as a rough estimation. The estimated power level for the DP seems approximately on the order of the power threshold for FAIs observed by Wright et al. (2006).

4. The dependence of the SBS and DP lines on aspect angle of the transmitter beam relative to the magnetic field $\theta_{0}$ is different. The SBS occurs where the parallel electric field undergoes swelling at the plasma resonance altitude. The DP requires a large electric field component perpendicular to the geomagnetic field in the $\mathrm{UH}$ region. When increasing $\theta_{0}$ for the pump wave, the parallel electric field component becomes smaller and the electric field turns from parallel to the geomagnetic field towards horizontal at a larger distance below the reflection height (Leyser, 1991). For tilting beam angles off the magnetic field, the excitation of SBS from the reflection region becomes relatively less important compared to SBS from the upper hybrid level (Fu et al., 2013). For varying beam angle experiments, strong DP emissions were essentially observed for the magnetic zenith beam (Tereshchenko et al., 2006).

5. Both SBS and DP are associated with IA waves that depend on electron temperature. As electron temperature, $T_{\mathrm{e}}$, is increased during heating, the frequency offsets of SBS and DP are predicted to increase. If $f_{0}$ is sufficiently far from $3 f_{c e}$, the electron temperature may be derived based on IA SBS emission from the upper hybrid resonance level (Bernhardt et al., 2009). Electron temperature retrieval from SBS and DP lines is a potentially powerful diagnostic capability; however important aspects of the theory are still lacking and more work is required at this time.

Finally, it should be pointed out that both EISCAT $(\Theta \approx$ $\left.12^{\circ}\right)$ and HAARP $\left(\Theta \approx 14^{\circ}\right)$ HF heating facilities are located at high latitudes with a comparable geomagnetic angle. The EISCAT HF heater has approximately one-third the power of the HARRP HF heater and only higher gyro-harmonic $(n \geq 3)$ heating capability is available at EISCAT. However, with the unique advantage of the EISCAT/UHF radars and CUTLASS radars, new SEE phenomena recently observed at HAARP may be investigated in further detail at EISCAT as well.

Acknowledgements. The authors would like to acknowledge Ingemar Häggström for access to EISCAT UHF radar data analysis and the staff at the EISCAT facility for technical support. The work at Virginia Tech was supported in part by the National Science Foundation. The work at the Naval Research Laboratory was sponsored by the NRL 6.1 Base programme. The work at Fudan University was supported in part by National Science Foundation of China (NSFC no. 41404122). The authors highly appreciate the constructive comments from the referees.

The topical editor K. Hosokawa thanks S. Grach and T. Leyser for help in evaluating this paper.

\section{References}

Bernhardt, P. A., Selcher, C. A., Lehmberg, R. H., Rodriguez, S., Thomason, J., McCarrick, M., and Frazer, G.: Determination of the electron temperature in the modified ionosphere over HAARP using the HF pumped Stimulated Brillouin Scatter (SBS) emission lines, Ann. Geophys., 27, 4409-4427, doi:10.5194/angeo-27-4409-2009, 2009.

Bernhardt, P. A., Selcher, C. A., Lehmberg, R. H. , Rodriguez, S. P., Thomason, J. F. , Groves, K. M. , McCarrick, M. J., and Frazer, G. J.: Stimulated Brillouin Scatter in a magnetized ionospheric plasma, Phys. Rev. Lett., 104, 165004, 2010.

Dysthe, K. B., Leer, E., Trulsen, J., and Stenflo, L.: Stimulated Brillouin scattering in the ionosphere, J. Geophys. Res., 82, 717-718, 1977. 
Dysthe, K. B., Mjølhus, E., Pecsel, H. L., and Rypdal, K.: A thermal oscillating two-stream instability, Phys. Fluids, 26, 146-157, 1983.

Fejer, J. A.: Stimulated Brillouin scattering and incoherent backscatter, J. Geophys. Res., 4, 289-290, 1977.

Fejer, J. A., Rinnert K., and Woodman R.: Detection of stimulated Brillouin scattering by the Jicamarca radar, J. Geophys. Res., 83, 2133-2136, 1978.

Fialer, P. A.: Field-aligned scattering from a heated region of the ionosphere - Observations at HF and VHF, Radio Sci., 9, 923940, 1974.

Fu, H., Scales, W. A., Bernhardt, P. A., Samimi, A., Mahmoudian, A., Briczinski, S. J., and McCarrick, M. J. : Stimulated Brillouin scatter and stimulated ion Bernstein scatter during electron gyroharmonic heating experiments, Radio Sci., 48, 607-616, 2013.

Honary, F., Stocker, A. J., Robinson, T. R., Jones, T. B., and Stubbe, P.: Ionospheric plasma response to $\mathrm{HF}$ radio waves operating at frequencies close to the third harmonic of the electron gyrofrequency, J. Geophys. Res., 100, 21489-21501, 1995.

Honary, F., Robinson, T. R., Wright, D. M., Stocker, A. J., Rietveld, M. T., and McCrea, I.: Letter to the Editor: First direct observations of the reduced striations at pump frequencies close to the electron gyroharmonics, Ann. Geophys., 17, 1235-1238, doi:10.1007/s00585-999-1235-6, 1999.

Huang, J. and Kuo, S. P.: A generation mechanism for the downshifted peak in stimulated electromagnetic emission spectrum, J. Geophys. Res., 100, 21433-21438, 1995.

Hussein, A. A. and Scales, W. A.: Simulation studies of parametric decay processes assosiated with ionospheric stimulated radiation, Radio Sci., 32, 2099-2107, 1997.

Kosch, M. J., Rietveld, M. T., Kavanagh, A. J., Davis, C., Yeoman, T. K., Honary, F., and Hagfors, T.: High-latitude pump-induced optical emissions for frequencies close to the third electron gyroharmonic, Geophys. Res. Lett., 29, 27-1-27-4, 2002.

Kruer, W. L.: The Physics of Laser Plasma Interactions, AddisonWesley, New York, USA, 1988.

Leyser, T. B.: Parametric interaction between upper hybrid and lower hybrid waves in heating experiments, Geophys. Res. Lett., 18, 408-411, 1991.

Leyser, T. B., Thidé, B., Kopka, H., Waldenvik, M., Veszelei, E, Frolov, V. L., Grach, A. M., and Komrakov, G. P.: Downshifted maximum features in stimulated electromagnetic emission spectra, J. Geophys. Res., 99, 19555-19568, 1994.

Leyser, T. B.: Stimulated electromagnetic emissions by highfrequency electromagnetic pumping of the ionospheric plasma, Space Sci. Rev., 98, 223-328, 2001.

Mahmoudian, A., Scales, W. A., Bernhardt, P. A., Samimi, A., Kendall, E., Ruohoniemi, J. M., Isham, B., Vega-Cancel, O., and Bordikar, M.: Ion gyro-harmonic structuring in the stimulated radiation spectrum and optical emissions during electron gyro-harmonic heating, J. Geophys. Res., 118, 1270-1287, doi:10.1002/jgra.50167, 2013a.

Mahmoudian, A., Scales, W. A., Bernhardt, P. A., Fu, H., Briczinski, S. J., and McCarrick, M. J. : Investigation of ionospheric stimulated Brillouin scatter generated at pump frequencies near electron gyroharmonics, Radio Sci., 48, 685-697, 2013b.

Norin, L., Leyser, T. B., Nordblad, E., Thidé, B. and McCarrick, M.: Unprecedentedly strong and narrow electromagnetic emissions stimulated by high-frequency radio waves in the ionosphere, Phys. Rev. Lett., 102, 065003, doi:10.1103/PhysRevLett.102.065003, 2009.

Pedersen, T., Gustavsson, B., Mishin, E., Kendall, E., Mills, T., Carlson, H. C., and Snyder, A. L.: Creation of artificial ionospheric layers using high-power HF waves, Geophys. Res. Lett., 37, L02106, doi:10.1029/2009GL041895, 2010.

Rietveld, M. T., Kohl, H., Kopka, H., and Stubbe, P.: Introduction to iono-spheric heating experiments at Troms $\varnothing, 1$, Experimental overview, J. Atmos. Terr. Phys., 55, 577-599, 1993.

Robinson, T. R., Stocker, A. J., Bond, G. E., Eglitis, P., Wright, D. M., and Jones, T. B.: O- and X-mode heating effects observed simultaneously with the CUTLASS and EISCAT radars and low power HF diagnostics at Troms $\varnothing$, Ann. Geophys., 15, 134-136, 1997 , http://www.ann-geophys.net/15/134/1997/.

Samimi, A., Scales, W. A., Bernhardt, P. A., Briczinski, S. J., Selcher, C. A., and McCarrick, M. J.: On ion gyro-harmonic structuring in the stimulated electromagnetic emission spectrum during second electron gyro-harmonic heating, Ann. Geophys., 30, 1587-1594, doi:10.5194/angeo-30-1587-2012, 2012.

Samimi, A., Scales, W. A., Fu, H., Bernhardt, P. A., Briczinski, S. J., and McCarrick, M. J.: Ion gyroharmonic structures in stimulated radiation during second electron gyroharmonic heating: 1. Theory, J. Geophys. Res., 118, 502-514, 2013.

Samimi, A., Scales, W. A., Bernhardt, P. A., Briczinski, S. J., and McCarrick, M. J.: Ion gyroharmonic structures in stimulated radiation during second electron gyroharmonic heating: 1. Simulations, J. Geophys. Res., 119, 502514, doi:10.1002/2013JA019341, 2014.

Stubbe, P. and Kopka, H.: Stimulated electromagnetic emission in a magnetized plasma: A new symmetric spectral feature, Phys. Rev. Lett., 65, 183-186, 1990.

Stubbe, P., Stocker, A. J., Honary, F., Robinson, T. R., and Jones, T. B.: Stimulated electromagnetic emissions and anamalous HF wave absorption near electron gyroharmonics, J. Geophys. Res., 99, 6233-6246, 1994.

Stubbe, P., Kopka, H., Thidé, B., and Derblom, H.: Stimulated electromagnetic emission: A new technique to study the parametric decay instability in the ionosphere, J. Geophys. Res.-Atmos., 89, 7523-7536, 1984.

Tereshchenko, E. D., Yurik, R. Yu., Khudukon, B. Z., Rietveld, M. T., Isham, B., Belyey, V., Brekke, A., Hagfors, T., and Grill, M.: Directional features of the downshifted peak observed in HF-induced stimulated electromagnetic emission spectra obtained using an interferometer, Ann. Geophys., 24, 1819-1827, doi:10.5194/angeo-24-1819-2006, 2006.

Thidé, B., Kopka, H., and Stubbe, P.: Observations of stimulated scattering of a strong high-frequency radio wave in the ionosphere, Phys. Rev. Lett., 49, 1561-1564, 1982.

Thidé, B., Hedberg, A., Fejer, J. A., and Sulzer, M. P. : First observations of stimulated electromagnetic emission at Arecibo, Geophys. Res. Lett., 16, 369-372, 1989.

Wright, D. M., Davies, J. A., Yeoman, T. K., Robinson, T. R., and Shergill, H.: Saturation and hysteresis effects in ionospheric modification experiments observed by the CUTLASS and EISCAT radars, Ann. Geophys., 24, 543-553, doi:10.5194/angeo24-543-2006, 2006. 